\title{
Reducing Hospital Lengths of Stay by Discharge Status
}

\author{
Ronald Lagoe $^{1 *}$, Suzanne Marra ${ }^{2}$, Lisa Crawford ${ }^{3}$, Barbara Drapola ${ }^{4}$ \\ ${ }^{1}$ Hospital Executive Council, Syracuse, NY, USA \\ ${ }^{2}$ St. Joseph's Hospital Health Center, Syracuse, NY, USA \\ ${ }^{3}$ Upstate University Hospital, Syracuse, NY, USA \\ ${ }^{4}$ Crouse Hospital, Syracuse, NY, USA \\ Email: ^Hospexcl@cnymail.com
}

How to cite this paper: Lagoe, R., Marra, S., Crawford, L. and Drapola, B. (2019) Reducing Hospital Lengths of Stay by Discharge Status. Case Reports in Clinical Medicine, 8, 1-8.

https://doi.org/10.4236/crcm.2019.81001

Received: December 11, 2018

Accepted: January 6, 2019

Published: January 9, 2019

Copyright (C) 2019 by author(s) and Scientific Research Publishing Inc. This work is licensed under the Creative Commons Attribution International License (CC BY 4.0).

http://creativecommons.org/licenses/by/4.0/

\section{(c) (i) Open Access}

\begin{abstract}
This study evaluated the impact of length of stay reduction by discharge status in the hospitals of Syracuse, New York. It focused on the two largest inpatient services, adult medicine and adult surgery, between 2008 and 2018. In the Syracuse hospitals, the adult medicine mean length of stay declined by 0.12 days, resulting in a savings of 14,154 patient days during the ten year period. The adult surgery mean stays declined by 0.91 days, resulting in a savings of 22,639 patient days. The reductions in stays were accompanied by differences in utilization by discharge status. For discharges to self care, the changes in mean stays increased the number of days saved for adult medicine from 5111 to 13,264 and the number of days saved for adult surgery from 4355 to 13,862 . These changes were brought about through internal efficiencies within the hospitals. For discharges to nursing homes, the reductions in stays caused the number of excess days to decline from 13,631 to 8695 for adult medicine and from 9150 to 5075 for adult surgery. These changes were brought about through cooperative efforts with long term care providers in the community.
\end{abstract}

\section{Keywords}

Hospitals, Hospital Admissions, Health Care Expenses

\section{Introduction}

In the United States, efforts are continuing to focus on improving the efficiency of health care. The expenses of health care have become a major burden for government through Medicare and Medicaid, as well as for businesses and individuals through private insurance. In recent years, efforts to address this issue have, 
at best, slowed the rate of increase of these expenses [1] [2].

Although health care payers operate at national and regional levels, the delivery of care is carried out at the local level. Most communities support acute care, ambulatory care, and long term care resources. Both the availability and use of these resources determine the outcomes of health care and the costs of producing them [3] [4].

From the standpoint of utilization, improving the efficiency of hospitals and other health care providers involves reducing inpatient admissions and lengths of stay. Reducing admissions is essential for health care payers because it is directly associated with reimbursement, but reducing stays is more important for providers because it is directly related to outcomes and internal expenses [5] [6].

Within health care at the community level, hospitals provide acute episodic care. As a result, hospital care is followed by other modalities such as long term care, ambulatory care, or informal supports. For many patients, the efficiency of acute care depends on the effectiveness of the other types of care in the continuum [7] [8].

It has been demonstrated that reducing hospital inpatient lengths of stay frequently has a positive impact on outcomes. It lowers the possibility of post admission complications and other adverse events [9].

Reducing stays has a major impact on hospital efficiency. By expediting the movement of patients to other levels of care, it reduces the expenses of inpatient hospitalization.

\section{Population}

This study described the impact of reducing inpatient hospital lengths of stay by discharge status in the metropolitan area of Syracuse, New York during a 10 year period. This area includes three large acute care facilities, Crouse Hospital (18,935 inpatient discharges excluding well newborns-2017), St. Joseph's Hospital Health Center (24,886 inpatient discharges-2017), and Upstate University Hospital (32,411 inpatient discharges-2017).

The hospitals provide primary and secondary acute care to a service area with a population of approximately 600,000 . They also provide a full range of services to the eleven county Central New York Health Service Area with a population of $1,400,000$.

Historically, the Syracuse hospitals have worked cooperatively to improve the efficiency of care in the service area. They have maintained an inpatient admission rate per capita that is the lowest of the metropolitan areas in New York State. They have also implemented programs that have contributed to reductions in inpatient lengths of stay [10].

\section{Method}

This study focused on inpatient lengths of stay by discharge status for the combined Syracuse hospitals between 2008 and 2018. It included stays for adult 
medicine and adult surgery, the inpatient services with the largest discharge volumes.

Within these services, the study identified hospital stays by discharge status. This indicator included discharges to self care (home without organized services), nursing home (skilled nursing facilities), home health care (home through certified home health agencies), expired and interhospital transfers.

Reductions in hospital stays for discharges to self care were implemented through programs within the hospitals because organized post discharge services were not required. The need for these programs was evaluated through monitoring of the times needed to deliver inpatient acute care services. For adult medicine, these programs included expediting the provision of diagnostic tests and medication regimes. For adult surgery, these programs included early ambulation of patients after procedures and expediting the provision of therapies. This discharge status usually involved relatively large numbers of discharges and short stays per patient.

Reductions in hospital stays for discharges to nursing homes were implemented through programs that moved patients at the end of their stays to post discharge services in the community. For adult medicine and adult surgery, these programs included the identification and monitoring of difficult to place patients in all of the hospitals. They also included the implementation of subacute programs involving long term acute care services such as intravenous antibiotic therapy and extensive wound care in nursing homes. These subacute and complex care programs were developed through cooperation with long term care providers. These programs usually involved relatively limited numbers of discharges and long stays per patient.

The utilization data for the combined hospitals were identified with simple descriptive statistics for each discharge status and hospital service by even numbered years. Numbers of discharges were also identified for each year.

The annual mean lengths of stay for the combined hospitals were compared with the severity adjusted national average stays for each discharge status and inpatient service. Severity adjusted national averages were identified using the All Patients Refined Diagnosis Related Group System developed by $3 \mathrm{M}^{\mathrm{rm}}$ Health Information Services. The differences between hospital stays and national average stays were multiplied by numbers of hospital discharges to identified patient days differences. Positive differences indicated hospital stays above national averages. Negative differences indicated hospital stays below national averages.

The impact of the reductions in hospital stays for adult medicine and adult surgery were estimated in utilization, including numbers of patient days and average daily censuses eliminated for the hospitals. They were also quantified as the estimated expenses of elimination of this utilization.

\section{Results}

The first part of the study focused on length of stay reduction in the Syracuse 
hospitals for adult medicine patients. Related data are summarized in Table 1.

The lengths of stay data for adult medicine were affected by the implementation of medical observation regulations for Medicare patients in October 2013. As a result of these regulations, a number of Medicare patients were moved from inpatient to observation status. Consistent with that change, the study data identify a reduction in discharges and an increase in mean stays for adult medicine in the Syracuse hospitals beginning in 2014.

The data in Table 1 demonstrated that the total mean length of stay for adult medicine in the Syracuse hospitals declined by 0.12 days, from 4.98 to 4.86 days, between 2008 and 2018. This produced a change from 8843 excess days to 5311 days saved annually, resulting in the elimination of 14,154 patient days and an average daily census of 38.8 , compared with severity adjusted national averages. The data also demonstrated that, since implementation of the medicare observation regulations, the adult medicine mean length of stay declined by 0.58 days, from 5.44 to 4.86 days between 2014 and 2018 .

The study data demonstrated that the largest numbers of patients for the Syracuse hospitals involved discharges to self care. This population involved 14,603 - 19,247 patients during the period of the study. As a result of programs that improved efficiency within the hospitals, mean length of stay for this population declined from 3.53 to 3.47 days between 2008 and 2018. This change increased the number of days saved annually from 5111 to 13,264 . The data also demonstrated that after the implementation of the Medicare observation regulations, the mean length of stay for discharges to self care increased from 4.18 to 4.27 days because patients at lower severity of illness had been moved to observation status.

The data in Table 1 demonstrated that the largest numbers of adult medicine patients with excess stays in the Syracuse hospitals were those discharged to nursing homes. Between 2008 and 2018, the mean length of stay for these patients increased from 7.40 to 7.62 days. After implementation of the medical observation program by Medicare and initiatives involving the hospitals and area nursing homes, the mean length of stay declined by 1.26 days, from 8.88 to 7.62. This resulted in a reduction in the number of excess days for this population from 13,631 to 8695 , an average daily census of 13.5 .

The adult medicine data also indicated that the reduction of hospital stays for discharges to home care decreased the number of excess days for this discharge status from 5191 to 1326 and increased the number of days saved for deaths/transfers from 542 to 2176 during the period of the study. These efficiencies were related to hospital wide efforts to reduce lengths of stay, rather than specific programs.

The second part of the study focused on reduction of hospital stays for adult surgery patients in the Syracuse hospitals. Related data are summarized in Table 2.

Adult surgery patients were not involved with medical observation, so the continuum of utilization for this service was consistent between 2008 and 2018 . 
The data in Table 2 demonstrated that, during this period, the mean length of stay for adult surgery patients declined by 0.91 days, from 6.23 to 5.32 days, between 2008 and 2018. This resulted in a change from 11,724 excess days to 10,915 days saved, eliminating 22,639 patient days and an average daily census of 62.0 days in the hospitals.

The study data demonstrated that the largest numbers of adult surgery patients were discharged home with self care. This discharge status accounted for $10,000-12,500$ patients annually. As a result of the implementation of programs that improved efficiency within the hospitals, the mean length of stay for this population declined from 3.53 to 3.05 days between 2008 and 2018. This change increased the annual number of days saved from 4355 to 13,862 .

The study data also demonstrated that the largest numbers of patients with excess stays in the Syracuse hospitals were those discharged to nursing homes. Between 2008 and 2018, the mean length of stay for these patients increased from 10.50 to 10.58 days. During this period, the severity of illness of these patients increased at a rate higher than the severity adjusted national average. This resulted in a decline in the number of excess days for this discharge status from 9150 to 5075 .

Table 1. Inpatient adult medicine mean lengths of stay by discharge status, Syracuse hospitals, 2008-2018.

\begin{tabular}{|c|c|c|c|c|c|c|}
\hline & Jan-Dec 2008 & Jan-Dec 2010 & Jan-Dec 2012 & Jan-Dec 2014 & Jan-Dec 2016 & Jan-Oct 2018 \\
\hline \multicolumn{7}{|c|}{ Number of Discharges } \\
\hline Self Care & 14,603 & 17,752 & 19,247 & 18,152 & 17,574 & 16,581 \\
\hline Home Care & 6409 & 6368 & 7655 & 6867 & 6543 & 6630 \\
\hline Nursing Home & 4932 & 5056 & 5008 & 4957 & 5434 & 4969 \\
\hline Deaths/Transfers & 2583 & 3067 & 3339 & 3443 & 3478 & 3066 \\
\hline Total & 28,527 & 32,243 & 35,249 & 33,419 & 33,029 & 31,246 \\
\hline \multicolumn{7}{|c|}{ Mean Lengths of Stay } \\
\hline Self Care & 3.53 & 3.60 & 3.62 & 3.81 & 3.61 & 3.47 \\
\hline Home Care & 5.84 & 6.38 & 6.24 & 6.40 & 5.81 & 5.70 \\
\hline Nursing Home & 7.40 & 8.20 & 8.31 & 8.88 & 7.80 & 7.62 \\
\hline Deaths/Transfers & 6.43 & 6.83 & 6.56 & 7.21 & 5.97 & 6.11 \\
\hline Total & 4.98 & 5.18 & 5.14 & 5.44 & 4.98 & 4.86 \\
\hline \multicolumn{7}{|c|}{ Patient Days Difference } \\
\hline Self Care & -5111.05 & -7366.36 & -10778.32 & -10528.16 & -11247.36 & -13264.80 \\
\hline Home Care & 5191.29 & 6559.04 & 5511.60 & 4669.56 & 2617.20 & 1326.00 \\
\hline Nursing Home & 9420.12 & 11932.16 & 11318.08 & 13631.75 & 11248.38 & 8695.75 \\
\hline Deaths/Transfers & -542.43 & 184.02 & -1435.77 & 378.73 & -2399.82 & -2176.86 \\
\hline Total & 8843.37 & 11285.05 & 4934.86 & 8020.56 & 0.00 & -5311.82 \\
\hline
\end{tabular}

Source: Hospital Executive Council. 
Table 2. Inpatient adult surgery mean lengths of stay by discharge status, Syracuse hospitals, 2008-2018.

\begin{tabular}{|c|c|c|c|c|c|c|}
\hline & Jan-Dec 2008 & Jan-Dec 2010 & Jan-Dec 2012 & Jan-Dec 2014 & Jan-Dec 2016 & Jan-Oct 2018 \\
\hline \multicolumn{7}{|c|}{ Number of Discharges } \\
\hline Self Care & 10,370 & 10,108 & 11,091 & 11,048 & 12,439 & 11,090 \\
\hline Home Care & 4533 & 4830 & 5104 & 5108 & 5436 & 3563 \\
\hline Nursing Home & 3081 & 3010 & 3122 & 3170 & 3377 & 2476 \\
\hline Deaths/Transfers & 1236 & 1220 & 1089 & 1231 & 1433 & 1064 \\
\hline Total & 19,220 & 19,168 & 20,406 & 20,557 & 22,685 & 18,193 \\
\hline \multicolumn{7}{|c|}{ Mean Lengths of Stay } \\
\hline Self Care & 3.53 & 3.55 & 3.38 & 3.56 & 3.40 & 3.05 \\
\hline Home Care & 7.14 & 7.41 & 7.37 & 6.75 & 6.53 & 6.45 \\
\hline Nursing Home & 10.50 & 10.02 & 10.29 & 10.18 & 10.88 & 10.58 \\
\hline Deaths/Transfers & 14.97 & 14.69 & 14.77 & 14.74 & 12.66 & 13.05 \\
\hline Total & 6.23 & 6.25 & 6.04 & 6.04 & 5.85 & 5.32 \\
\hline \multicolumn{7}{|c|}{ Patient Days Difference } \\
\hline Self Care & -4355.40 & -5963.72 & -8096.43 & -9280.32 & -12065.83 & -13862.50 \\
\hline Home Care & 3218.43 & 2994.60 & 3623.84 & 1123.76 & 380.52 & -1817.13 \\
\hline Nursing Home & 9150.57 & 7314.30 & 8242.08 & 8115.20 & 8712.66 & 5075.80 \\
\hline Deaths/Transfers & 3757.44 & 2452.20 & 2232.45 & 2068.08 & -1146.40 & -159.60 \\
\hline Total & 11724.20 & 6900.48 & 5917.74 & 2055.70 & -4083.30 & -10915.80 \\
\hline
\end{tabular}

Source: Hospital Executive Council.

The adult surgery data also indicated that the reduction of hospital stays for discharges to home care increased the number of days saved for this discharge status from 3218 excess days to 1817 days saved and increased the number of days saved for deaths/transfers from 3757 excess days to 159 days saved during the period of the study. These efficiencies were related to hospital wide efforts to reduce lengths of stay, rather than specific programs.

\section{Discussion}

This study evaluated the impact of inpatient length of stay reduction by discharge status in the hospitals of Syracuse, New York between 2008 and 2018. It focused on differences in stays and related utilization for the two largest services, adult medicine and adult surgery.

This study and the programs it addressed were based on the use of severity of illness data for inpatient lengths of stay in the hospitals. These data enabled the hospital staffs to identify length of stay issues within adult medicine and adult surgery and address them. Without severity of illness data, decreases or increases in stays might have resulted from variations in the characteristics of the inpatient populations, rather than the impact of length of stay reductions.

At the aggregate level, the study demonstrated that stays declined for both 
services during the ten year period. The adult medicine mean length of stay declined by 0.12 days, resulting in a savings of 14,154 patient days. At a late stay expense of $\$ 600$ per patient day, this would amount to approximately $\$ 8,500,000$ in annual expenses. The adult surgery mean length of stay declined by 0.91 days, resulting in a savings of 22,639 patient days. At the same rate, this would amount to $\$ 13,500,000$ in annual expenses.

The reductions in stays were accompanied by different changes in utilization by discharge status. For discharges to self care, the changes in mean stays were 0.06 days for adult medicine and 0.48 days for adult surgery. For this discharge status, discharge volumes were large, resulting 12,000 - 13,000 annual days saved per year. These reductions were achieved through efficiencies within hospitals. They were supported by hospital control over their own organizations.

For discharges to nursing homes, there were limited increases in actual mean stays that were offset by increased severity of illness. Length of stay reduction for this discharge status was more challenging because of the need to work with long term care providers in the community. For this discharge status, excess days remained at the end of the ten year period.

The study excluded discharges to home health care, deaths, and inter hospital transfers because they were not addressed by specific programs in the hospitals. At the end of the period, mean stays for adult surgery patients discharged to home care, as well as deaths and transfers for both services were shorter than severity adjusted national averages.

The study demonstrated the uses of data related to hospital discharge status in the planning and implementation of length of stay reduction at the community level. Further research can help identify additional uses of this information.

\section{Conflicts of Interest}

The authors declare no conflicts of interest regarding the publication of this paper.

\section{References}

[1] Dentzler, S. (2011) Urgent Measures for an Old Problem. Health Affairs, 30, 1626. https://doi.org/10.1377/hlthaff.2011.0961

[2] Meyer, H. (2018) The Price Problem. Modern Healthcare, 48, 20-24.

[3] Shah, B.R., Reed, S.D., Francis, J., Ridley, D.B. and Schulman, K.A. (2003) The Cost of Inefficiency in U.S. Hospitals, 1985-1987. Journal of Health Care Finance, 30, $1-9$.

[4] Pena, A.D. and Ndiyae, M. (2002) Developing Hospital Efficiency Cost Control Measures. World Hospitals and Health Services, 38, 41-43.

[5] Cushing, W.T. (2004) Extra Hospital Days Can Cost You Plenty. Medical Economics, 7, 81-83.

[6] Friedman, B., De La Mare, J., Andrews, R. and McKenzie, D.H. (2002) Practical Options for Estimating the Costs of Hospital Stays. Journal of Health Care Finance, 291, 1-12. 
[7] Oriol, W. (1985) The Complex Cube of Long Term Care. American Health Planning Association, Washington DC.

[8] Anderson, G. and Knickman, J.R. (2001) Changing the Chronic Care System to Meet Peoples' Needs. Health Affairs, 20, 146-160. https://doi.org/10.1377/hlthaff.20.6.146

[9] Weil, A.R. (2015) Hospital Cost and Quality. Health Affairs, 34, 1263. https://doi.org/10.1377/hlthaff.2015.0786

[10] Lagoe, R., Pasinski, T., Kronenberg, P., Quinn, T. and Schaengold, P. (2006) Linking Health Services at the Community Level. Canada Healthcare Quarterly, 9 , 60-65. https://doi.org/10.12927/hcq..18229 